\title{
One-dimensional Continuum Electronic Structure with the Density Matrix Renormalization Group and Its Implications for Density Functional Theory
}

\author{
E.M. Stoudenmire, Lucas O. Wagner, Steven R. White, and Kieron Burke \\ Department of Physics and Astronomy, University of California, Irvine, CA $926 \overrightarrow{97}$
}

(Dated: October 26, 2018)

\begin{abstract}
We extend the density matrix renormalization group to compute exact ground states of continuum many-electron systems in one dimension with long-range interactions. We find the exact ground state of a chain of 100 strongly correlated artificial hydrogen atoms. The method can be used to simulate $1 \mathrm{~d}$ cold atom systems and to study density functional theory in an exact setting. To illustrate, we find an interacting, extended system which is an insulator but whose Kohn-Sham system is metallic.
\end{abstract}

PACS numbers: 71.15.Dx, 31.15.-p, 05.10.Cc, 71.15.Mb, 31.15.E-

For electronic structure calculations, these are the best of times and the worst of times. When correlations are weak, density functional theory (DFT) makes it possible to tackle extremely realistic Hamiltonians and large system sizes with reasonable accuracy [1]. For strongly correlated systems, there exist powerful and controllable numerical methods 2 for simulating lattice Hamiltonians, such as the Hubbard model. However, few numerical tools can treat the combination of strongly correlated electronic systems and realistic microscopic Hamiltonians. In the strongly correlated regime, DFT approximations are neither systematic nor controllable, often leading to unrestrained parameter multiplication and empiricism. Model Hamiltonians rely on the arbitrary truncation of terms that may be crucial in tipping the balance between competing phases. Attempts to bridge the gap between realistic Hamiltonians and strong correlation techniques, such as dynamical mean field theory coupled to DFT [3, 4, may contain both arbitrary truncations and a less than ideal treatment of correlations.

Therefore we would like to study DFT in an exact setting to see how density functional approximations break down and whether new approximations contain the right physics. But very few continuum, three-dimensional, long-range interacting systems can be easily treated exactly. Here, we show that by studying one dimensional (1d) systems instead, we can treat realistic Hamiltonians and strong electron correlations essentially exactly, even for a very large number of atoms. Because they preserve the continuum, our 1d models mimic key features of three-dimensional reality surprisingly well 5 .

Our approach is based on the density matrix renormalization group (DMRG) 6], the most powerful of the strongly correlated techniques for 1d lattice models. Here we extend DMRG to treat continuum electron systems with long-range interactions. This new approach retains DMRG's exponential convergence and near linear scaling with system size. As an example, we present a near exact calculation of a system with 100 strongly interacting pseudo-hydrogen atoms (Fig. 1).

A key motivation for this method is to study DFT in an exact setting, both when correlations are strong and

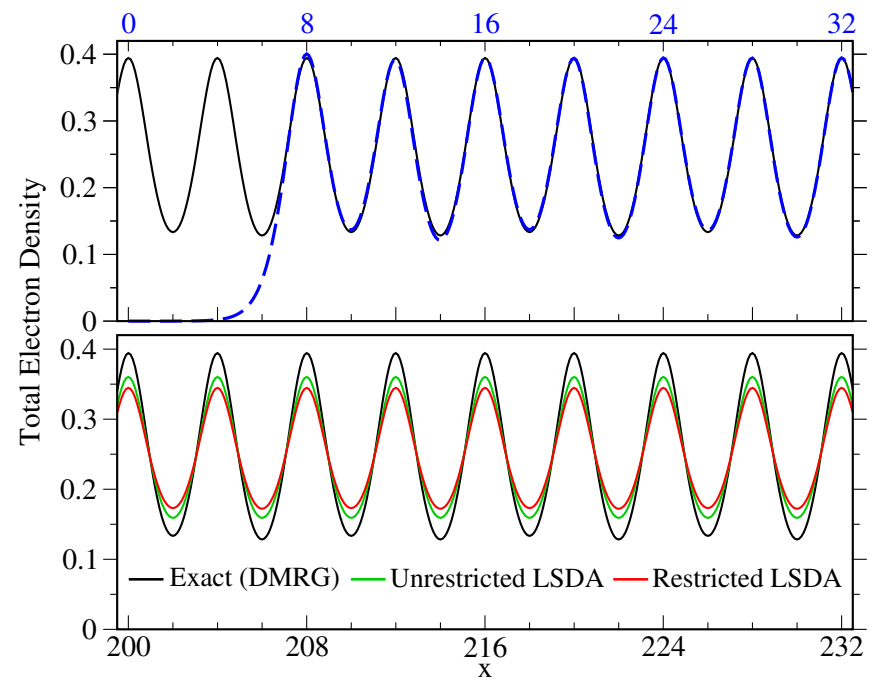

FIG. 1. The exact ground state density of a chain of 100 widely separated (strongly correlated) artificial atoms. The total length of the system is $L=420$ in atomic units (4200 grid sites with a spacing of 0.1 ). The upper panel shows the electron density of a central region superimposed with the density at the left edge (the dashed blue curve with corresponding $x$ above). The lower panel compares the exact electron density to DFT predictions within the local spin density approximation.

near the thermodynamic limit. Generically, 1d systems have strong quantum fluctuations, making them an especially rigorous test of DFT approximations; they can also be pushed to large size with less effort. As in Fig. 1, we can easily compare various DFT approximations with exact results for extended systems. We can also compute exact quantities appearing in the DFT formalism; for example, we show below that a gapped interacting system can nevertheless have a Kohn-Sham gap which is exactly zero (a Mott insulator [7]). DMRG also offers new ways to characterize electronic structure models using quantum information concepts, such as the bipartite entanglement entropy. Finally, 1d continuum Hamiltonians can be realized exactly in cold atom systems [8, 9].

Presently, real-space DMRG methods for solid-state applications are designed only to work with lattice mod- 
els. Each site of such a model can be thought of as a Wannier function centered on an atom. One way of generalizing this picture to make the Hamiltonian more realistic is to expand in a set of basis functions; DMRG has become a powerful technique for the quantum chemistry of small molecules based on this idea [10, 11. Here we proceed in a more flexible direction which does not depend on a choice of basis and has optimal scaling of calculation time with system size: we represent the continuum in $1 \mathrm{~d}$ with a real space grid. The continuum Hamiltonians of interest can be written as

$$
\begin{aligned}
H & =\sum_{\sigma} \int_{x} \psi_{\sigma}^{\dagger}(x)\left[-\frac{1}{2} \frac{\partial^{2}}{\partial x^{2}}-\mu\right] \psi_{\sigma}(x) \\
& +\int_{x} v(x) n(x)+\frac{1}{2} \int_{x, x^{\prime}} v_{\mathrm{ee}}\left(x-x^{\prime}\right) n(x) n\left(x^{\prime}\right) .
\end{aligned}
$$

where $\psi_{\sigma}^{\dagger}(x)$ creates an electron of spin $\sigma$ at position $x ; v$ and $v_{\text {ee }}$ are local and electron-electron potentials, respectively; and $n$ is the electron density operator. We introduce a grid spacing $a$, obtaining a discretized Hamiltonian

$$
\begin{aligned}
H & =\sum_{j, \sigma} \frac{-1}{2 a^{2}}\left(c_{j \sigma}^{\dagger} c_{j+1 \sigma}+c_{j+1 \sigma}^{\dagger} c_{j \sigma}\right)-\tilde{\mu} n_{j \sigma} \\
& +\sum_{j} v^{j} n_{j}+\frac{1}{2} \sum_{i, j} v_{\mathrm{ee}}^{i j} n_{i}\left(n_{j}-\delta_{i j}\right)
\end{aligned}
$$

where $\tilde{\mu}=\mu-1 / a^{2}, v^{j}=v(j a)$ and $v_{\mathrm{ee}}^{i j}=v_{\mathrm{ee}}(|i-j| a)$. The $\delta_{i j}$ in the last term prevents an unphysical self interaction. For technical reasons we work with open boundaries rather than periodic, and extend the grid well past the edge atoms. Finite grid spacing errors can be reduced arbitrarily by reducing $a$; convergence can be accelerated by using a higher order discretized derivative [5]. Here, we fix $a=0.1$ in atomic units.

A different approach was proposed recently by Verstraete and Cirac [12, who showed how to define and optimize matrix product states directly in the continuum limit in the context of quantum field theories.

Working efficiently with such a Hamiltonian represents an unusual challenge for DMRG. Normally, one is most concerned with the number of states per block $m$ needed to represent the ground state. The number of sweeps $N_{S}$ needed to converge to the ground state is usually quite small $(\sim 2-5)$ for $1 \mathrm{~d}$ systems. Here, the reverse can happen: a small grid spacing ' $a$ ' relative to the interatomic separation can lead to energy scales that differ by orders of magnitude, greatly increasing $N_{S}$ but not affecting $m$ significantly. Fortunately, while convergence with $m$ reflects the entanglement of the system, an inherent property, many approaches can be tried to reduce $N_{S}$. We have found a particularly efficient acceleration approach based on a real-space RG procedure which produces a supplementary grid with a much coarser spacing and lower energy scales such that $N_{S}$ can be made small; after sweeping on this grid we map the wave function back onto the fine grid for further sweeps. This procedure, which we hope to discuss in a future publication, introduces no additional approximations; it merely reduces the computational time to reach a converged ground state.

Besides the wide range of energy scales, another challenge for DMRG is the presence of long-ranged interactions. The simplest approach, dealing with $N_{g}^{2}$ terms in a sweep over $N_{g}$ grid points, would scale as $N_{g}^{3}$. A more efficient approach using intermediate operators, as used with DMRG for quantum chemistry in a basis set, would scale as $N_{g}^{2}$. We utilize a much more efficient approach than either of these by using a representation of the Hamiltonian as a matrix product operator (MPO). Finite bond dimension MPOs naturally encode exponentially decaying interactions [13. Interactions with a power law decay can be approximated to high accuracy by fitting to a sum of $N_{\mathrm{MPO}}$ exponentials (usually $N_{\mathrm{MPO}} \leq 50$ is sufficient to obtain an accuracy of $10^{-5}$ ) [14. Thus the DMRG calculation time is nominally linear in $N_{g}$; specifically, it is proportional to $N_{S} N_{g} N_{\mathrm{MPO}} \mathrm{m}^{3}$. Deviations from a purely linear computational effort with the overall system size can come from a dependence of $N_{S}$ or $m$ with system size; in practice we find that the RG acceleration procedure keeps $N_{S}$ small. The behavior of $m$ is well understood for short-ranged model Hamiltonians: for noncritical systems, $m$ is independent of system size. For critical systems, with power-law decaying correlations, $m$ grows only logarithmically with length. Hence, we expect only a slightly worse-than-linear computational effort with system size in the worst case.

As a simple setting for exploring strong many-body correlation effects, we consider chains of one-dimensional "soft hydrogen" atoms. Each atom consists of a single electron in a soft-Coulomb potential well of the form

$$
v_{\text {atom }}(x)=-1 / \sqrt{x^{2}+1},
$$

and we use atomic units throughout. We also include repulsive interactions defined by $v_{\text {ee }}\left(x-x^{\prime}\right)=-v_{\text {atom }}\left(\left|x-x^{\prime}\right|\right)$. Using bare Coulomb interactions would lead to an ill-defined model in 1d; this potential is a standard choice for avoiding such complications and has been used to study molecules in intense laser fields [15, 16]. We are also fortunate to benefit from the work of Ref. 17, which provides a parameterization of the $1 \mathrm{~d}$ local spin density approximation (LSDA) for just such an interaction. We note that by using state-of-the-art DMRG it is also possible to simulate a chain of real hydrogen atoms [18]. But our purely $1 \mathrm{~d}$ setting allows us to study a wider variety of systems and, in the future, explore dynamical and finite-temperature effects.

To demonstrate of the power of our approach, we display in Fig. 1 the exact ground state density of a chain of one hundred artificial atoms with long-range interactions, which took a few days of computer time on a single 
workstation. Representing the ground state accurately required keeping about $m=200$ states. The relative energy error in the many-body solution from DMRG is of order $10^{-6}$. The relative errors due to the finite grid spacing and finite number of exponentials $N_{\mathrm{MPO}}$ used to fit $v_{\mathrm{ee}}$ are larger, of order $10^{-4}$, but are well understood and easily reduced if necessary. Also shown in Fig. 1 1 are DFT calculations within both the restricted and unrestricted LSDA [17. For this system, both DFT approaches make substantial errors. In terms of lattice models, one would represent this system with either a half-filled Hubbard chain or an antiferromagnetic Heisenberg chain. Both models are critical with power law decaying spin-spin correlations; a noncritical system would have been easier for DMRG. It is not surprising that the local DFT approximations cannot capture the quasi-long-range spin correlations, with the unrestricted LSDA predicting longrange antiferromagnetism (similar to Fig. 2). It is somewhat surprising that even the total density from LSDA deviates strongly from the exact DMRG results. The total energy from each LSDA calculation is off by about $1 \%$.

In Fig. 2 we show a system which reveals weaknesses of both approximate DFT and of model Hamiltonian approaches. The figure shows the exact ground state density of ten atoms with interatomic spacing $b=4$. The edges induce a staggered pattern of strong and weak bonds which decays slowly into the bulk, and is therefore significant throughout this small system. We can understand the staggered behavior from a 10 site Hubbard model (at half filling with $U / t=4$ chosen arbitrarily) or a 10 site Heisenberg model. The Heisenberg ground state has resonating valence bond character; in a perfect near-neighbor RVB state, the edges would suppress all resonance and drive the weak bonds to zero. The actual Heisenberg ground state has longer range resonances which reduce these effects. In the Hubbard model, the strong exchange bonds show up as bonds with lower kinetic energy. However, neither lattice model reveals the increased electron density on the strong bonds, stemming from the strong hopping. These models might be improved by bond-dependent interactions $t$ and $J$. The LSDA calculations capture even fewer properties of the true ground state. Unrestricted LSD predicts an energy -11.364 which is close to the exact energy -11.496 , but no staggered bond density and breaks spin symmetry, producing a long-ranged antiferromagnetic state as shown in the lower panel of Fig. 2. Restricted LSDA captures the staggered density pattern qualitatively, but gives a slightly higher energy -11.323 and fails to reproduce the correct local spin correlations since its wave function is a Slater determinant of extended orbitals. The artificial symmetry breaking of LSDA can be understood as a frozen spin fluctuation [19], but the exact functional yields a singlet ground state.

Not only can we compare our exact results to DFT approximations, but we can use them to investigate fundamental questions about DFT itself. The fundamental (charge) gap is $E_{g}=(I-A)$ where $I$ is the ionization potential and $A$ the electron affinity. In Fig. 3, we compute $E_{g}$ for chains of soft hydrogen atoms with spacing $b=4$ for large systems up to $N=60$ atoms $(\sim 2500$ grid sites). Extrapolation shows the $N \rightarrow \infty$ system to be an insulator. We also compute the exact Kohn-Sham (KS) gap for each $N$ by inverting the density of the neutral system to obtain the KS potential and its single particle energies. (Given an interacting system, the KS system is the unique non-interacting system with the same density [20.) In the thermodynamic limit, the KS gaps extrapolate to zero, so that the exact $N \rightarrow \infty \mathrm{KS}$ system is a metal. This is consistent with the fact that each finite KS system in Fig. 3 has one electron per unit cell and thus


FIG. 2. Spontaneous dimerization of the density for a chain of 10 soft hydrogen atoms with interatomic spacing $b=4$ (dashed lines are a guide to the eye). The upper panel compares the densities predicted by DFT within the LSDA; the lower panel shows the spin densities for unrestricted LSDA. Also shown is the expectation value of the kinetic energy $\left\langle c_{j}^{\dagger} c_{j+1}+c_{j+1}^{\dagger} c_{j}\right\rangle$ for a Hubbard model with $U / t=4$ and the exchange energy $\left\langle\mathbf{S}_{j} \cdot \mathbf{S}_{j+1}\right\rangle$ for the Heisenberg model on 10 lattice sites. The thickness of the lines indicates the magnitude of these quantities on each bond. 


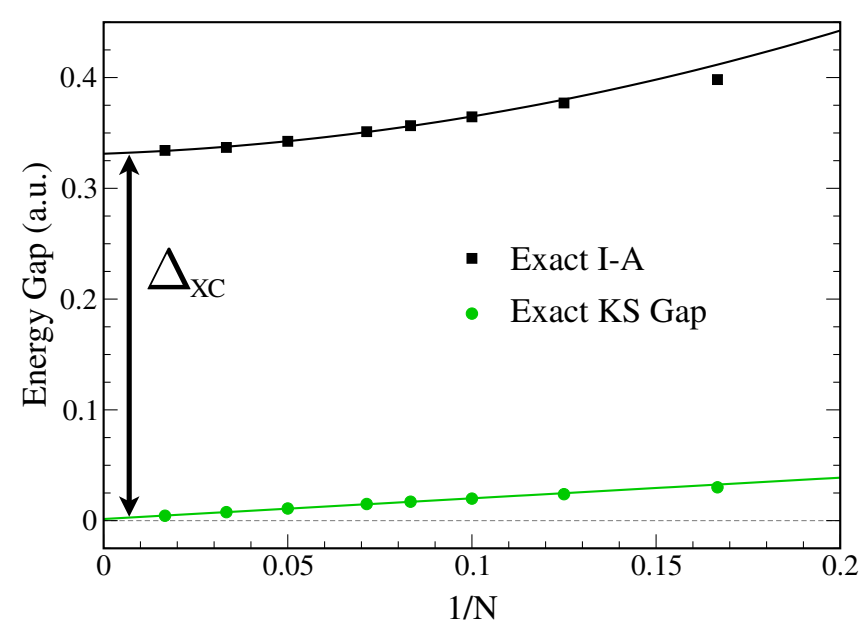

FIG. 3. Exact gaps for chains of $N$ soft hydrogen atoms with atomic separation $b=4$ (error bars are less than symbol sizes). The upper curve is a quadratic fit of exact gaps of the largest six systems and extrapolates to a finite value $E_{g} \simeq 0.33$. The exact Kohn-Sham gaps, in contrast, extrapolate to zero showing that for $N \rightarrow \infty$ the true KS system is metallic (lower curve is a linear fit of exact KS gaps of the largest six systems).

a half-filled band (in contrast to the unrestricted LSDA which breaks spin symmetry for this system).

The discrepancy between the KS and exact gap was long ago identified [21] with the exchange-correlation derivative discontinuity in DFT: $E_{g}=\Delta_{s}+\Delta_{\mathrm{XC}}$ where $\Delta_{s}$ is the KS gap, that is, the HOMO-LUMO energy difference for the neutral KS system. Approximate functionals such as LSDA that are continuous in particle number miss this effect entirely. The LSDA KS gaps are almost identical to the exact ones shown in Fig. 3 , but the LSDA fundamental gap drops from close to $E_{g}$ for small $N$ to near zero at large $N$ (details reported elsewhere).

Previous calculations have found $\Delta_{\mathrm{XC}}$ for semiconductors [22, 23] with finite KS gaps $\Delta_{s}$, but our system's gap is entirely due to $\Delta_{\mathrm{XC}}$, underscoring its importance for strong correlation physics. Our results rely on no uncontrolled approximations and so demonstrate unambiguously the behavior of Mott insulators in DFT. Present DFT research on this issue focuses on extracting accurate $E_{g}$ from semilocal functional calculations [24, 25].

The onset of strong correlation with increasing bond length is often identified with the Coulson-Fischer point [26], where an unrestricted Hartree-Fock calculation spontaneously breaks spin symmetry. A different way to distinguish strong from weak correlation is through the entanglement spectrum, readily accessible in DMRG. Defining the left reduced density matrix $\rho_{L}=\operatorname{Tr}_{R}|\Psi\rangle\langle\Psi|$, where the trace is over all grid sites in the right half of the system, the entanglement spectrum consists of the energies of the entanglement Hamiltonian $H_{E}=-\ln \rho_{L}$ [27]. The most probable density matrix eigenstates are those

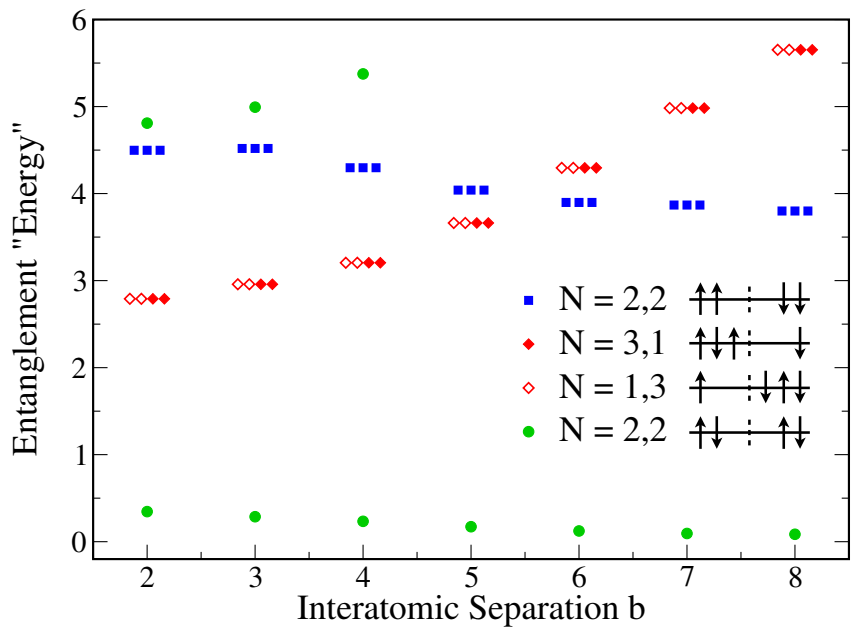

FIG. 4. Entanglement spectrum at the center of interacting 4-atom chains with various interatomic separations $b$. $N=\left(N_{L}, N_{R}\right)$ refers to the number of electrons to the left and right of the cut for each density matrix eigenstate. The states with $N_{L}=3,1$ primarily correspond to charge fluctuations while those with $N_{L}=2$ to spin fluctuations.

in the low "energy" part of the spectrum. By classifying these states according to their particle number $N_{L}$, we can understand the dominant quantum fluctuations of the ground state. Figure 4 shows the entanglement spectrum at the center of a series of four-atom chains with increasing interatomic separation. A sharp crossover at $b \simeq 5.5$, where the probability for charge fluctuations drops below that of pure spin fluctuations, signals the onset of strongly correlated behavior.

Many oxide materials of current interest are too strongly correlated for present DFT methods, but crucial properties must be calculated to an accuracy far beyond that of simple model Hamiltonians. The method described here provides a new, alternative route to studying strongly correlated systems. All existing approximations, from heuristic corrections to standard functionals, such as $\mathrm{LDA}+\mathrm{U} 28$, to methods developed for lattice models, such as dynamical mean field theory [29], can be applied and tested more easily, thoroughly, and accurately in the present setting. Because our 1d world captures a feature crucial to density functional approximations, namely the continuum instead of a lattice, such studies should provide the insight needed to construct more accurate density functionals for real strongly-correlated materials.

Note added: After completing this Letter, we became aware of Ref. 30 which is similar in spirit to our real-space RG method for accelerating continuum DMRG.

We gratefully acknowledge DOE grant DE-FG0208ER46496 (KB, LW and SW) and NSF grant DMR0907500 (ES and SW) for supporting this work. 
* Also at Department of Chemistry, University of California, Irvine, CA 92697

[1] R. M. Martin, Electronic structure: Basic theory and practical methods (Cambridge University Press, 2004); C. Fiolhais, F. Nogueira, and M. Marques, eds., $A$ Primer in Density Functional Theory (Springer-Verlag, NY, 2003).

[2] P. Corboz, S. R. White, G. Vidal, and M. Troyer, Phys. Rev. B, 84, 041108 (2011); A. M. Läuchli, J. Sudan, and E. S. Sørensen, Phys. Rev. B, 83, 212401 (2011) A. W. Sandvik, Phys. Rev. Lett., 104, 177201 (2010).

[3] V. Anisimov, A. Poteryaev, M. Korotin, A. Anokhin, and G. Kotliar, Journal of Physics: Condensed Matter, 9, 7359 (1997)

[4] A. I. Lichtenstein and M. I. Katsnelson, Phys. Rev. B, $\mathbf{5 7 ,} 6884(1998)$

[5] L. O. Wagner, E. M. Stoudenmire, K. Burke, and S. R. White, Phys. Chem. Chem. Phys., 14, 7559 (2012).

[6] S. R. White, Phys. Rev. Lett., 69, 2863 (1992) Phys. Rev. B, 48, 10345 (1993)

[7] N. F. Mott and R. Peierls, Proc. Phys. Soc., 49, 72 (1937) N. F. Mott, Proc. Phys. Soc. A, 62, 416 (1949)

[8] L. Jiang, T. Kitagawa, J. Alicea, A. R. Akhmerov, D. Pekker, G. Refael, J. I. Cirac, E. Demler, M. D. Lukin, and P. Zoller, Phys. Rev. Lett., 106, 220402 (2011)

[9] M. A. Cazalilla, R. Citro, T. Giamarchi, E. Orignac, and M. Rigol, Rev. Mod. Phys., 83, 1405 (2011)

[10] S. R. White and R. L. Martin, J. Chem. Phys., 110, 4127 (1999).

[11] G. K.-L. Chan and S. Sharma, Annual Review of Physical Chemistry, 62, 465 (2011).

[12] F. Verstraete and J. Cirac, Phys. Rev. Lett., 104, 190405 (2010)
[13] I. P. McCulloch, J. Stat. Mech., P10014 (2007)

[14] B. Pirvu, V. Murg, J. I. Cirac, and F. Verstraete, New J. Phys., 12, 025012 (2010).

[15] J. Eberly, Q. Su, and J. Javanainen, J. Opt. Soc. Am. B, 6, 1289 (1989)

[16] M. Thiele, E. K. U. Gross, and S. Kümmel, Phys. Rev. Lett., 100, 153004 (2008).

[17] N. Helbig, J. I. Fuks, M. Casula, M. J. Verstraete, M. A. L. Marques, I. V. Tokatly, and A. Rubio, Phys. Rev. A, 83, 032503 (2011).

[18] J. Hachmann, W. Cardoen, and G. K.-L. Chan, The Journal of Chemical Physics, 125, 144101 (2006).

[19] J. P. Perdew, A. Savin, and K. Burke, Phys. Rev. A, 51, 4531 (1995).

[20] W. Kohn and L. J. Sham, Phys. Rev., 140, A1133 (1965).

[21] J. P. Perdew, R. G. Parr, M. Levy, and J. L. Balduz, Phys. Rev. Lett., 49, 1691 (1982).

[22] W. Knorr and R. W. Godby, Phys. Rev. Lett., 68, 639 (1992); Phys. Rev. B, 50, 1779 (1994)

[23] M. Grüning, A. Marini, and A. Rubio, Phys. Rev. B, 74, $161103(2006)$.

[24] M. K. Y. Chan and G. Ceder, Phys. Rev. Lett., 105, $196403(2010)$

[25] X. Zheng, A. J. Cohen, P. Mori-Sánchez, X. Hu, and W. Yang, Phys. Rev. Lett., 107, 026403 (2011)

[26] C. Coulson and I. Fischer, Philosophical Magazine Series 7, 40, $386(1949)$

[27] A. M. Turner, F. Pollmann, and E. Berg, Phys. Rev. B, 83, $075102(2011)$.

[28] V. I. Anisimov, J. Zaanen, and O. K. Andersen, Phys. Rev. B., 44, 943 (1991)

[29] A. Georges, G. Kotliar, W. Krauth, and M. J. Rozenberg, Rev. Mod. Phys., 68, 13 (1996)

[30] M. Dolfi, B. Bauer, M. Troyer, and Z. Ristivojevic, Phys. Rev. Lett., 109, 020604 (2012) 